\title{
Substrate Turnover by Transporters Curtails Synaptic Glutamate Transients
}

\author{
Steven Mennerick, ${ }^{1}$ Weixing Shen, ${ }^{1}$ Wanyan Xu, ${ }^{1}$ Ann Benz,, ${ }^{1}$ Kohichi Tanaka, ${ }^{3}$ Keiko Shimamoto, ${ }^{4}$ \\ Keith E. Isenberg, ${ }^{1}$ James E. Krause, ${ }^{2}$ and Charles F. Zorumski ${ }^{1,2}$ \\ Departments of ${ }^{1}$ Psychiatry and ${ }^{2}$ Anatomy and Neurobiology, Washington University School of Medicine, St. Louis, \\ Missouri 63110, ${ }^{3}$ Department of Molecular Neuroscience, Medical Research Institute, Tokyo Medical and Dental \\ University, Bunkyo-Ku, Tokyo 113-8519, Japan, and ${ }^{4}$ Suntory Institute for Bioorganic Research, Wakayamadai, \\ Shimamoto-cho, Mishima-gun, Osaka 618, Japan
}

\begin{abstract}
Although inhibitors of glutamate transport prolong synaptic currents at many glutamate synapses, the cause of the current prolongation is unclear. Transport inhibitors may prolong synaptic currents by simply interfering with synaptic glutamate binding to transporters, by inhibiting substrate translocation, or by promoting accumulation of ambient glutamate, which may act cooperatively at receptors with synaptic glutamate. We show that reversal of the membrane potential of astrocytes surrounding the synapse prolongs synaptic currents but does not decrease the apparent affinity of transporters or significantly alter glutamate-dependent kinetics of macroscopic transporter currents in excised membrane patches. Positive membrane potentials do not affect binding of a nontransported glutamate analog, nor do positive membrane potentials alter the number of transporters available to bind analog. We also test the hy-
\end{abstract}

pothesis that glutamate accumulation during uptake inhibition by transporter substrates is the direct cause of synaptic current prolongations. Transporter substrates elevate ambient glutamate near synapses by fostering reverse transport of endogenous glutamate. However, increases in ambient glutamate cannot account for the prolongations of synaptic currents, because a nonsubstrate transport inhibitor does not foster reverse uptake yet it prolongs synaptic currents. Moreover, exogenous glutamate does not mimic synaptic current prolongations induced by substrate inhibitors. These results provide strong support for a major role of substrate translocation in determining the time course of the glutamate concentration transient at excitatory synapses.

Key words: glutamate; postsynaptic; uptake; transporter; EPSC; desensitization
Understanding the factors that dictate the time course of glutamate's actions at CNS synapses may help elucidate glutamate's double-edged role of neurotransmitter and neurotoxin. Recent experiments have revealed that pharmacological blockade of glutamate transporters increases the peak glutamate concentration in the synaptic cleft (Tong and Jahr, 1994; Diamond and Jahr, 1997) or prolongs the glutamate transient, leading to a slowed EPSC decay at some synapses (Barbour et al., 1994; Mennerick and Zorumski, 1994, 1995; Takahashi et al., 1995; Otis et al., 1996; Kinney et al., 1997). Slowed EPSC decays have been observed at a number of glutamate synapses in the presence of transport inhibitors, but the precise transporter action responsible for truncating synaptic glutamate transients remains incompletely understood.

Besides blocking endogenous substrate (glutamate) translocation from the extracellular to intracellular compartment, uptake inhibitors have two other actions that could account for prolonged synaptic currents. Based on turnover rates in the $10 / \mathrm{sec}$ range (three orders of magnitude slower than acetylcholinesterase at

Received June 30, 1999; revised Aug. 19, 1999; accepted Aug. 20, 1999.

This work was supported by National Institutes of Health Grants MH-00964 and MH-45493 and a gift from the Bantley Foundation (C.F.Z.) and a Lucille P. Markey postdoctoral fellowship (S.M.). We thank Joe Tucker, Amy Zarrin, Yuki Izumi, and Bob Cormier for discussion, and Aguan Wei for the gift of the pOX expression vector.

Correspondence should be addressed to Dr. Steven Mennerick, Department of Psychiatry, Washington University School of Medicine, 4940 Children's Place, St. Louis, MO 63110. E-mail: menneris@psychiatry.wustl.edu.

Copyright (C) 1999 Society for Neuroscience 0270-6474/99/199242-10\$05.00/0 the neuromuscular junction), it has been suggested that transporters are too slow to transport a significant amount of glutamate to the intracellular compartment on the millisecond time scale of synaptic transmission (Lester et al., 1994; Wadiche et al., 1995b; Wadiche and Kavanaugh, 1998). Therefore, it has been hypothesized that during individual synaptic events, glutamate transporters normally act as stationary buffers. Once bound, glutamate is more likely to dissociate than to be transported (Wadiche and Kavanaugh, 1998), but on dissociation from the transporter, levels of glutamate are too low to activate a significant number of receptors. By preventing interaction of synaptic glutamate with the substrate binding site on the transporter, exogenous substrates would prolong the postsynaptic actions of glutamate. We have shown that a nonpharmacological manipulation of glutamate transport, glial membrane depolarization, also prolongs synaptic currents (Mennerick and Zorumski, 1994, 1995). The effect of reversing glial membrane polarity is quantitatively similar to the actions of pharmacological uptake inhibitors (Mennerick and Zorumski, 1994). Although the buffering hypothesis is not mutually exclusive of the translocation hypothesis (Tong and Jahr, 1994; Diamond and Jahr, 1997), for the buffering hypothesis to entirely account for all observed effects on EPSCs, membrane depolarization should alter the glutamate binding properties of transporters, a prediction tested in the present work.

A second hypothesis to account for EPSC prolongations is that transport inhibitors promote ambient glutamate accumulation, which may itself prolong synaptic currents, analogous to cooperative interactions between exogenous transmitter and synaptic 
transmitter observed at the neuromuscular junction (Hartzell et al., 1975). Most glutamate uptake inhibitors are transporter substrates and are expected to promote extracellular glutamate accumulation through heteroexchange of intracellular glutamate during transporter cycling (Kanner and Bendahan, 1982). In addition, transport inhibition in some preparations causes accumulation of synaptic glutamate released from surrounding cells (Isaacson and Nicoll, 1993; Takahashi et al., 1995). We have shown that both glial depolarization and lithium substitution prolong synaptic currents at hippocampal microculture synapses, presumably by interfering with the voltage dependence and sodium dependence (Barbour et al., 1991) of glutamate transporter actions, but these treatments are also expected to promote increases in ambient glutamate (Szatkowski et al., 1990).

Here we use hippocampal microcultures and recombinant glutamate transporters to investigate the roles of glutamate binding, translocation, and heteroexchange in prolongations of synaptic currents. Using the recently identified ligand-gated ion channel properties of transporters, we show that membrane potential changes do not alter the binding properties of the transporter for glutamate and nontransported glutamate analogs. We also use the recently developed nonsubstrate transport inhibitor DL-threo- $\beta$ benzyloxyaspartate (TBOA) and low concentrations of exogenous glutamate to explore the possibility that accumulated glutamate might explain the effects of these transporter manipulations. We conclude that the prolongation of synaptic currents observed in hippocampal microcultures is attributable to a direct effect of inhibiting glutamate translocation rather than to an effect of buffering by transporters or to an indirect effect of accumulated glutamate.

\section{MATERIALS AND METHODS}

Microcultures. Hippocampal cells were prepared from 1-3 d postnatal Sprague Dawley rats and grown on microcultures as described previously (Mennerick et al., 1995). Briefly, slices of hippocampus 500-800 $\mu \mathrm{m}$ thick were incubated at $37^{\circ} \mathrm{C}$ with stirring in $1 \mathrm{mg} / \mathrm{ml}$ papain in oxygenated Leibovitz's L-15 medium. Single-cell suspensions were prepared by mechanical trituration in modified Eagle's medium supplemented with $5 \%$ horse serum, $5 \%$ fetal calf serum, $17 \mathrm{~mm}$ D-glucose, $400 \mu \mathrm{M}$ glutamine, $50 \mathrm{U} / \mathrm{ml}$ penicillin, and $50 \mu \mathrm{g} / \mathrm{ml}$ streptomycin. Cells were plated at $75 / \mathrm{mm}^{2}$ onto plastic culture dishes coated with a layer of $0.15 \%$ agarose and collagen droplets as described previously and incubated at $37^{\circ} \mathrm{C}$ in $5 \% \mathrm{CO}_{2}$. Cultures were treated with cytosine arabinoside $(5-10$ $\mu \mathrm{M})$ after $3 \mathrm{~d}$ in vitro and were used for experiments $4-14 \mathrm{~d}$ after plating.

Culture electrophysiology. Except where noted, the extracellular bath solution for whole-cell recording contained (in mM): $140 \mathrm{NaCl}, 4.0 \mathrm{KCl}$, 3.0 $\mathrm{CaCl}_{2}, 1.0 \mathrm{MgCl}_{2}, 10 \mathrm{HEPES}, 0.05 \mathrm{D}-\mathrm{APV}$. The standard whole-cell pipette solution for synaptic studies and for whole-cell glial recordings contained (in mM): 130 potassium gluconate, $4.0 \mathrm{NaCl}, 0.5 \mathrm{CaCl}_{2}, 5.0$ EGTA, and $10 \mathrm{HEPES}, 2.0 \mathrm{MgATP}_{2}$, and $0.5 \mathrm{GTP}$. For examination of neuronal responses to exogenous applications of drugs, the patch-pipette solution contained cesium methanesulphonate or cesium chloride in place of potassium gluconate, and nucleotide triphosphates were excluded. Other alterations of the extracellular and pipette solutions are as given in the text and figure legends. The $\mathrm{pH}$ of solutions was adjusted to 7.25. Whole-cell voltage-clamp recordings of excitatory autaptic currents were performed from solitary neurons using 1.5-4 $\mathrm{M} \Omega$ pipettes. Wholecell access resistance was compensated $90-100 \%$ using the compensation circuitry of an Axopatch 1-D patch-clamp amplifier (Axon Instruments, Foster City, CA). Neurons were stimulated with a $1.5 \mathrm{msec}$ voltage pulse to $0 \mathrm{mV}$ from a typical holding potential of $-70 \mathrm{mV}$. Extracellular solutions were exchanged with a gravity-driven local perfusion system consisting of six separate lines connected to a common delivery port. Solutions were delivered at a rate of $240 \mu \mathrm{l} / \mathrm{min}$ except where noted.

Oocyte expression. Coding regions of the mouse glutamate transporter GLAST were subcloned into the pOX expression vector, which contains $5^{\prime}$ and $3^{\prime}$ untranslated regions from the Xenopus $\beta$-globin gene to improve message stability (Shih et al., 1998). Transporter transcripts (15-50 ng) synthesized in vitro (Ambion mmessage mmachine) were injected into mature defolliculated oocytes. Oocytes were incubated in ND96 solution supplemented with $0.5 \mathrm{~mm}$ theophylline and $0.55 \mathrm{mg} / \mathrm{ml}$ pyruvate. The ND96 solution contained (in mM): $96 \mathrm{NaCl}, 2 \mathrm{KCl}, 1.8 \mathrm{CaCl}_{2}$, $1.0 \mathrm{MgCl}_{2}$, and $10 \mathrm{HEPES}$.

Oocyte electrophysiology. Oocytes were used for experiments $1-7 \mathrm{~d}$ after injection. Whole-cell transporter currents were recorded in twoelectrode voltage-clamp mode using a Warner OC $725 \mathrm{C}$ amplifier in virtual ground mode. Voltage-recording and current-injection electrodes were filled with $3 \mathrm{M} \mathrm{KCl}$ and had resistances of $\sim 1 \mathrm{M} \Omega$. Recordings were standardly performed in ND96 extracellular solution; alterations to this solution are as given in the text and figure legends. Solution exchanges on whole oocytes were achieved by placing the oocyte in a linear chamber (100 $\mu \mathrm{l}$ volume) and perfusing solutions from the common tip of a multibarrel pipette at a rate of $1.5 \mathrm{ml} / \mathrm{min}$. For patch recordings, oocytes were mechanically stripped of vitelline membrane after shrinking in hypertonic saline. For excised patch recordings, the extracellular solution contained $96 \mathrm{NaSCN}, 2.0 \mathrm{KCl}, 3.0 \mathrm{MgCl}_{2}, 10 \mathrm{HEPES}$, and the patchpipette solution contained (in mM): $100 \mathrm{KSCN}, 10 \mathrm{KCl}, 3 \mathrm{MgCl}_{2}, 10$ EGTA, 10 HEPES (Wadiche and Kavanaugh, 1998). Calcium was omitted from solutions to prevent contribution of an endogenous oocyte $\mathrm{Ca}^{2+}$-activated anion conductance to records. Rapid applications were made to outside-out membrane patches using a piezo element (Burleigh). On-rate and off-rate constants from excised patch currents were measured by fitting single exponential functions to the raw data beginning at $10 \%$ of the final current amplitude. An agar-bridge ground was used for all experiments in which extracellular chloride concentration was manipulated. Errors in text and figures represent SEM.

\section{RESULTS}

Our previous results have shown that inhibition of glutamate transporters by various pharmacological and nonpharmacological treatments prolong synaptic currents (Mennerick and Zorumski, 1994, 1995). In most cases, we used transport inhibitors in the presence of cyclothiazide, which we have proposed unmasks an effect of glutamate transport inihibitors by sensitizing AMPA receptors to residual glutamate (Mennerick and Zorumski, 1994). However, cyclothiazide may have other effects, such as presynaptic potentiation (Diamond and Jahr, 1995) or other undescribed effects. Therefore, we reexamined the effect of nonpharmacological inhibition of glutamate transporters on EPSCs, in the absence of cyclothiazide (CYZ). Figure $1 A$ shows that reversing the glial membrane potential, a nonpharmacological means of inhibiting transporters, prolongs some synaptic currents even in the absence of cyclothiazide. Consistent with our previous suggestion that transport inhibition under these conditions detectably alters the EPSC decay most in neurons with slow baseline EPSC decays, the average baseline 10-90\% decay time (24 $\pm 4 \mathrm{msec})$ was correlated with the effect of depolarizing underlying glia $(r=0.94)$ (Fig. 1). Over all 11 neurons, the increase in 10-90\% decay time was only $4 \pm 3 \mathrm{msec}$, although in the three cells with the slowest baseline decay $(43 \pm 9 \mathrm{msec})$, the increase in decay was $17 \pm 8$ msec. These results are very similar to our previous results using pharmacological transporter substrates (Mennerick and Zorumski, 1995). Figure $1 B$ shows an example in which reversal of the glial membrane potential had no detectable effect on the time course of an autaptic current. Also shown in Figure 1 are records of glial currents recorded at a membrane potential of $-70 \mathrm{mV}$. These currents are largely transporter-associated and may represent the electrogenic uptake (translocation) of glutamate into astrocytes (Mennerick and Zorumski, 1994). However, other ionic and capacitive currents not strictly linked to uptake are associated with transporter binding and activation (Wadiche et al., 1995a,b). Furthermore, it is not clear whether substrate translocation is responsible for the truncated EPSC when the astrocytes are clamped to $-70 \mathrm{mV}$. Therefore, although these results confirm our previous observations that glial depolarization can 


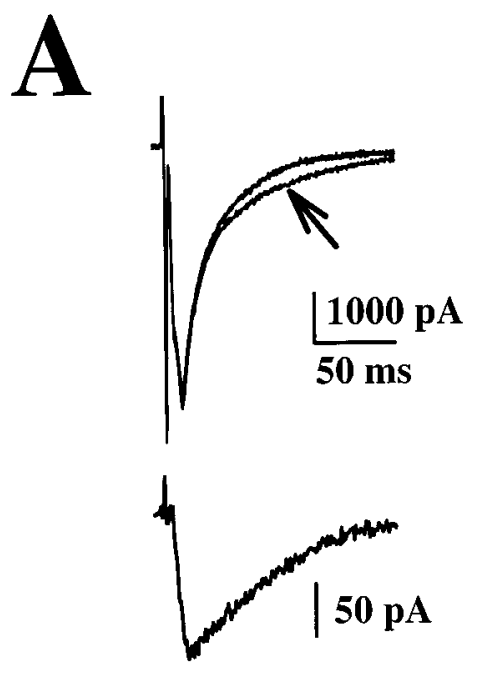

B
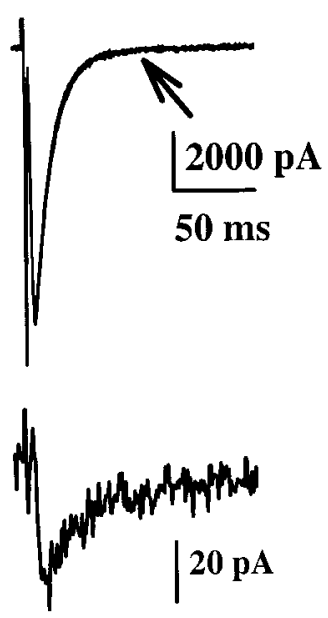

Figure 1. Reversal of glial membrane potential causes prolongation of synaptic current in some microcultures. $A$ (top traces), Autaptic currents from a solitary neuron in a hippocampal microculture. Six interleaved, superimposed traces represent two responses with underlying glia clamped at $-70 \mathrm{mV}$ command potential, two responses with glia clamped at $+60 \mathrm{mV} 20 \mathrm{sec}$ before stimulation (arrows), and two responses with recovery (glia clamped at $-70 \mathrm{mV}$ ). Bottom traces, Averaged response of the glia while clamped at $-70 \mathrm{mV}$. Glial responses at $+60 \mathrm{mV}$ were undetectable. $B$, Same protocol from a glia/neuron pair in another microculture in the same plate as the pair in $A$.

prolong EPSCs in the absence of cyclothiazide (Mennerick and Zorumski, 1995), they beg the question of whether substrate translocation is responsible for EPSC prolongations.

\section{Buffering by transporters cannot explain prolonged ESPCs}

Displacement of glutamate binding with competitive pharmacological inhibitors of transport may be sufficient to account for synaptic effects of transport inhibitors (Tong and Jahr, 1994; Diamond and Jahr, 1997; Wadiche and Kavanaugh, 1998). Given that manipulation of glial membrane potential has qualitatively effects similar to those observed with pharmacological uptake inhibitors, we sought to determine whether membrane depolar- ization alters glutamate binding to transporters. Because glutamate transporters also exhibit an anion conductance that, like transport itself, is gated by ligand binding to the transporter (Wadiche et al., 1995a), electrical currents in response to substrate are composed of both the electrogenic transport current and the anion current. To determine whether the transporter current and channel mode of the transporter share similar ligand concentration dependence, we coexpressed the GLAST glutamate transporter and $\mathrm{GABA}_{\mathrm{A}}$ receptors $(\alpha 1, \beta 2, \gamma 2 \mathrm{~L})$ in Xenopus oocytes. GABA responses were used to determine the chloride reversal potential $\left(E_{\mathrm{Cl}}\right)$ in oocytes $(-13 \pm 1.8 \mathrm{mV}, n=3)$. Next, glutamate concentration-response curves were obtained with oocytes voltage clamped at $E_{\mathrm{C} 1}$ to obtain a pure transport current. To estimate EC50 values, the data for individual cells were fit to the Hill equation (Fig. 2, legend). The $\mathrm{EC}_{50}$ for current generated at the chloride reversal potential did not differ significantly from the $\mathrm{EC}_{50}$ in the presence of $\mathrm{NO}_{3}{ }^{-}$, a condition in which anion flux should dominate the current $\left(35.9 \pm 2.9 \mu \mathrm{M}\right.$ at $E_{\mathrm{Cl},} n=6$ and $53.6 \pm 11 \mu \mathrm{M}$ in $\mathrm{NO}_{3}{ }^{-}, n=7$ oocytes; $\left.p>0.1\right)$. In addition, when $E_{\mathrm{C} 1}$ was shifted to $-40 \mathrm{mV}$ by overnight dialysis in $0 \mathrm{Cl}^{-}$saline, simlilar results were obtained $\left(\mathrm{EC}_{50}\right.$ values $41 \pm 7.2 \mu \mathrm{M}, n=6$ vs $29 \pm 4.7 \mu \mathrm{M}, n=5 ; p>0.1$ ). Average Hill coefficients for all conditions were similar and ranged from 1.2 to 1.4. Thus, the anion current and transport current show the same concentration dependence, and this is true at different voltages.

These results suggest that the ligand dependence of transport and anion flux through the channel mode of the transporter is similar, consistent with previous results (Wadiche et al., 1995a; Billups et al., 1996; Eliasof and Jahr, 1996). Therefore, we exploited the ligand-gated channel properties of the transporters to examine glutamate binding using techniques traditionally reserved for the examination of ligand-gated ion channels. First, we examined the effect of voltage on the apparent affinity of transporters for glutamate in Xenopus oocytes expressing glutamate transporters. Because we previously found that GLAST is the predominant transporter expressed in microculture glial cells (Mennerick et al., 1998), we examined recombinant GLAST expressed in oocytes. As shown in Figure 2, the apparent affinity for glutamate to gate the anion conductance was similar at +60 $\mathrm{mV}$ compared with $-80 \mathrm{mV}$. The $\mathrm{EC}_{50}$ fit to the average responses was $15.6 \mu \mathrm{M}$ at $+60 \mathrm{mV}$ versus $17.3 \mu \mathrm{M}$ at $-80 \mathrm{mV}$ in the same oocytes. The concentration-response curves were similar whether chloride or nitrate was used as a permeant anion (Fig. $2 B$ ), suggesting that manipulating the permeant anion does not influence the concentration-response relationship of the ligandgated current.

We performed similar experiments on native transporters in microculture astrocytes. To ensure good voltage clamp, we chose isolated, singly nucleated astrocyte islands for study. AMPA receptors were blocked with $5 \mu \mathrm{M}$ NBQX and $50 \mu \mathrm{M}$ GYKI 52466. Barium (6 $\mathrm{mm})$ replaced calcium and magnesium to diminish voltage-gated potassium conductances in the astrocytes. Transporter currents in native cells showed a somewhat higher apparent affinity for glutamate at positive potentials compared with negative potentials (Fig. $2 C$ ). From fits to average responses the $\mathrm{EC}_{50}$ was $10.0 \mu \mathrm{M}$ at $-90 \mathrm{mV}(n=3)$ and $1.7 \mu \mathrm{M}$ at $+60 \mathrm{mV}$ $(n=5)$.

Results from Figure 2 suggest that total binding of glutamate by transporters is not affected by membrane potential. However, examination of steady-state concentration-response relationships suffer from several limitations (Colquhoun, 1998), especially when drawing conclusions related to the nonequilibrium condi- 


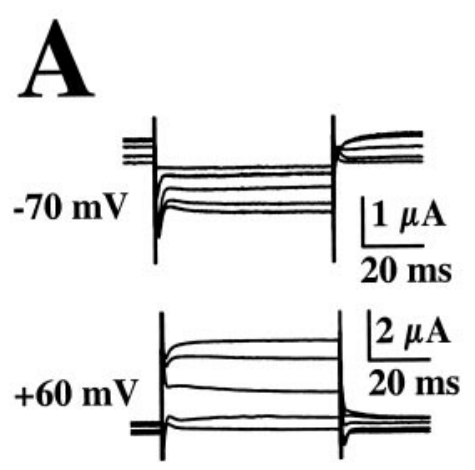

B

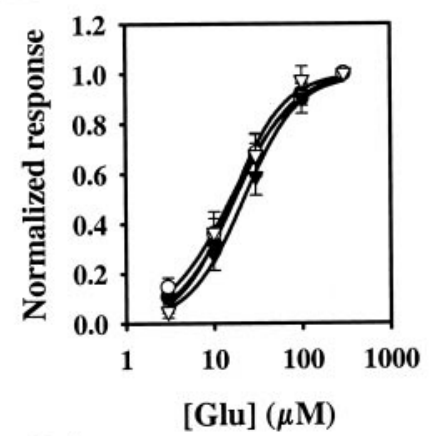

C
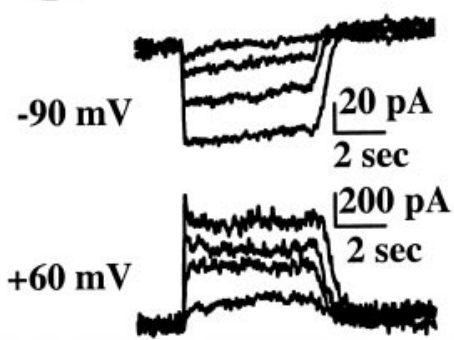

Figure 2. Steady-state concentration-response curves suggest a similar apparent affinity of the glial transporter GLAST for glutamate at negative and positive membrane potentials. $A$, Example traces from one oocyte obtained by stepping the membrane potential from -20 to $-70 \mathrm{mV}$ (top traces) or to $+60 \mathrm{mV}$ (bottom traces) in the absence and presence of glutamate. Ninety-three percent of extracellular chloride was replaced with $\mathrm{NO}_{3}{ }^{-}$. Control traces in the absence of glutamate were digitally subtracted from traces in the presence of glutamate. From smallest to largest, inward $(-70 \mathrm{mV})$ and outward currents $(+60 \mathrm{mV})$ represent responses to $3,10,30$, and $100 \mu \mathrm{M}$ glutamate. Because the traces represent subtractions, note that the change in current before the voltage jump represents the glutamateactivated currents at the holding potential of $-20 \mathrm{mV}$. B, Steady-state concentration-response curves measured in $\mathrm{NO}_{3}{ }^{-}$extracellular saline at $-80 \mathrm{mV}()_{)}$and $+60 \mathrm{mV}(\bigcirc)$ using the protocol in $A$ ( $n=6$ oocytes). Similar data were obtained in normal extracellular chloride at $-80 \mathrm{mV}(\mathbf{\Delta})$ and $+60 \mathrm{mV}(\triangle ; n=4$ oocytes $)$. Responses are normalized to the $300 \mu \mathrm{M}$ response in each condition. Solid lines represent fits to the Hill equation of the form $R=a[\mathrm{Glu}]^{\mathrm{b}} /\left(\mathrm{EC}_{50}{ }^{\mathrm{b}}+[\mathrm{Glu}]^{\mathrm{b}}\right)$ where $R$ is the response size, $a$ is the maximum response, and $\mathrm{EC}_{50}$ is the concentration generating halfmaximum response. $\mathrm{EC}_{50}$ values in the various conditions ranged from 15.6 to $22.9 \mu \mathrm{M}$. $C$, Responses of a cultured hippocampal astrocyte to varied glutamate concentrations at negative and positive membrane potentials. Glutamate was applied while holding the astrocyte at $-90 \mathrm{mV}$ (inward currents) and $+60 \mathrm{mV}$ (outward currents). $\mathrm{NaSCN}$ replaced $\mathrm{NaCl}$ in the extracellular solution. Responses were to $1,3,10$, and $100 \mu \mathrm{M}$ glutamate. The larger noise level at the positive membrane potential derives from unblocked voltage-gated conductances. tions of a synaptic glutamate pulse. One limitation is that the equilibrium concentration-response curves can be influenced by steps in the transport cycle other than the microscopic binding dissociation constant, including anion channel opening and closing rates (Colquhoun, 1998). Therefore, it is possible that voltage may alter binding to the transporter, but opposite compensatory changes in other kinetic steps cause a lack of shift in $\mathrm{EC}_{50}$ values. We addressed this problem by examining the influence of voltage on presteady-state currents isolated with varied concentrations of the nonsubstrate transport inhibitor TBOA. TBOA binds the glutamate binding site (Seal and Amara, 1998; Shimamoto et al., 1998) but is not transported. By binding the transporter, nonsubstrate analogs block capacitive charge movements that represent early steps in the transport cycle (Parent et al., 1992; Wadiche et al., 1995b). Thus, we used TBOA blockade of these capacitive currents to assess the voltage dependence of analog binding in the absence of late kinetic steps, including anion channel gating, in the transport cycle.

Figure $3 A, B$ shows that the TBOA concentration-response relationship is not altered by voltage. The absolute amplitude of instantaneous presteady-state current blocked by TBOA was used as a proportional measure of the number of transporters blocked by a given TBOA concentration. The instantaneous current showed the same concentration dependence whether TBOA was allowed to equilibrate with transporters at -80 or $+60 \mathrm{mV}$ (Fig. $3 A, B)$. This result strongly suggests that total binding of the glutamate analog TBOA is not affected by membrane potential. In addition, the $\mathrm{IC}_{50}$ for TBOA blockade of steady-state glutamate-gated transport currents was not affected by reversing membrane polarity (Fig. 3C,D), further suggesting no voltage dependence on total binding of either glutamate or TBOA (Colquhoun, 1998).

One possible explanation for the data in Figure $3 A, B$ is that when preapplied at a negative potential, TBOA is locked onto the transporter by a transporter conformational change during depolarization and that TBOA would be unable to bind the transporter if applied during depolarization. To test whether TBOA can bind the transporter at positive membrane potentials, we performed the experiment shown in Figure 4. In one protocol (Fig. 4A) $5 \mu \mathrm{M}$ TBOA was applied at $-70 \mathrm{mV}$ before a voltage pulse to $+60 \mathrm{mV}$. Subtraction of traces in the presence of TBOA from control traces in the absence of TBOA yielded symmetrical presteady-state currents (Fig. $4 A$ ) similar to those in Figure $3 A$. In a complementary protocol on the same oocyte, TBOA was applied $100 \mathrm{msec}$ after the onset of the pulse to $+60 \mathrm{mV}$. In this case, subtraction still yielded a negative-going presteady-state current on return to $-70 \mathrm{mV}$ that was similar in amplitude to the presteady-state current obtained in the standard protocol. In five oocytes the instantaneous current extrapolated from biexponential fits to data obtained from the protocol depicted in Figure $4 \mathrm{~A}$ was $118 \pm 21 \%$ of the current produced in the protocol in which TBOA was applied at $+60 \mathrm{mV}(p>0.1, n=5$ oocytes; paired $t$ test). As expected from this result, there were no differences in the time contants or amplitudes of the exponential fits to presteady-state current decay (at $-70 \mathrm{mV}$ ) in the two protocols ( $p>0.1, n=5$; paired $t$ tests). These results strongly suggest that equilibrium binding of a nonsubstrate glutamate analog is not voltage dependent.

Measurement of TBOA-sensitive presteady-state currents and glutamate currents are still equilibrium measurements of total binding and therefore may not reflect the nonequilibrium conditions occurring with synaptic release of glutamate. It is possible 

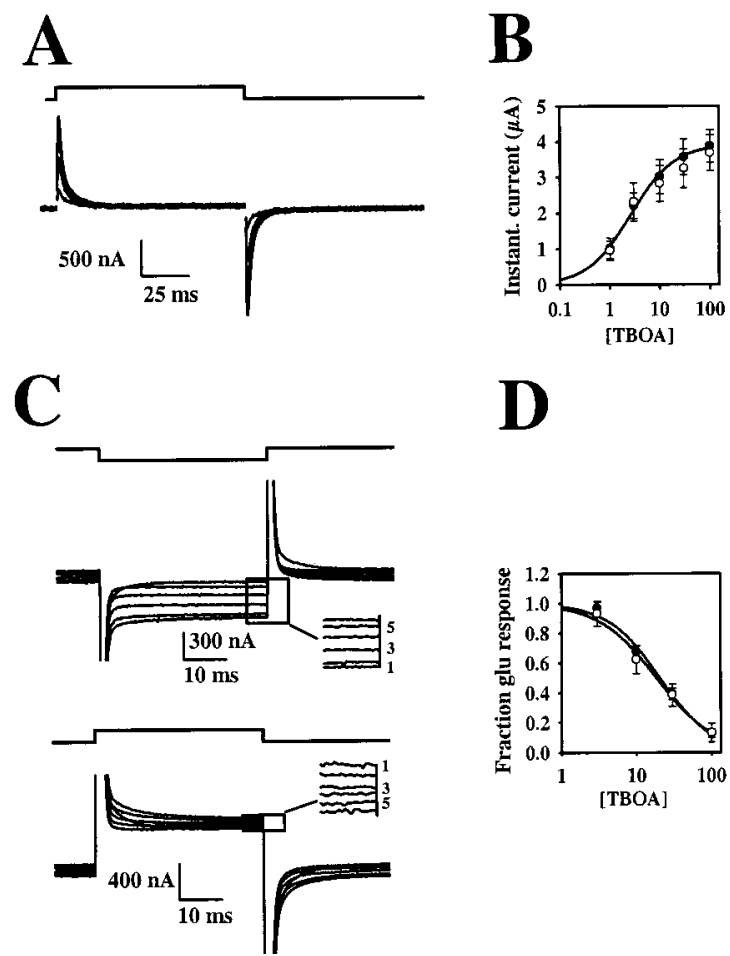

Figure 3. $\mathrm{EC}_{50}$ of a nonsubstrate glutamate analog is unchanged by altering the polarity of the membrane potential. $A$, Presteady-state currents (bottom traces) were isolated by pulsing the membrane potential of an oocyte expressing GLAST from -80 to $+60 \mathrm{mV}(100 \mathrm{msec})$ and then subtracting responses to the same protocol in varied concentrations of TBOA. From smallest to largest, the currents were isolated in 1, 3, 10, 30, and $100 \mu \mathrm{M}$ TBOA. $B$, Equilibrium concentration-response curve for the instantaneous current measured at $+60 \mathrm{mV}$ (after equilibration at -80 $\mathrm{mV}, 0$ ) and for the instantaneous currents measured at $-80 \mathrm{mV}$ (after equilibration at $+60 \mathrm{mV}, \bigcirc)$ in 13 oocytes. The polarity of the inward currents at $-80 \mathrm{mV}$ has been reversed and superimposed on the data at $+60 \mathrm{mV}$, but no normalization of current amplitudes was performed. The solid line represents a fit to the closed circles with an $\mathrm{EC}_{50}$ of $2.6 \mu \mathrm{M}$ and Hill coefficient of 1.0. C. The $\mathrm{IC}_{50}$ for inhibition of glutamate currents is not changed by reversing membrane potential polarity. Traces represent the current-response to a pulse of the membrane potential from -20 to $-80 \mathrm{mV}$ (top current traces) or to $+60 \mathrm{mV}$ (bottom current traces). Insets show the steady-state phase of the responses at a higher amplitude resolution. The voltage protocol was performed in the absence of drug (trace 6 ), in the presence of $20 \mu \mathrm{M}$ glutamate alone (trace 1), or glutamate plus 3, 10, 30, and $100 \mu \mathrm{M}$ TBOA (traces 2-5). For orientation, traces 1 , 3 , and 5 are labeled in both insets. $D$, The $\mathrm{IC}_{50}$ estimated from the fit to the inhibition data (solid line) was $20.4 \mu \mathrm{M}$ for $-80 \mathrm{mV}(\bullet)$ and $18.3 \mu \mathrm{M}$ at $+60 \mathrm{mV}(\bigcirc)$.

that although equilibrium binding is not affected by voltage, on and off rates of glutamate binding are each affected by an equal and opposite factor. For ligand-gated receptors, the binding rate constant of ligand can be estimated by examining the concentration dependence of current onset rate in response to step applications of low to moderate concentrations of ligand, where ligand binding is rate limiting (Lavoie and Twyman, 1996; Jones et al., 1998; Wadiche and Kavanaugh, 1998). Similarly, the deactivation rate of currents on the rapid removal of ligand are typically inversely correlated with agonist affinity (Lester and Jahr, 1992; Jones et al., 1998). Thus, to examine the effect of membrane voltage on transporter affinity more directly, we examined the on and off rates of currents in response to step applications of low to moderate glutamate concentrations (Fig. 5) in patches excised from oocytes, where expression levels were sufficient to observe

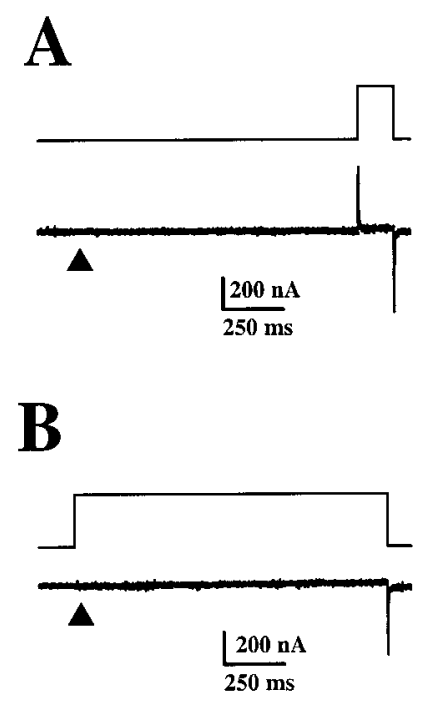

Figure 4. TBOA binds the transporter at positive membrane potentials. All data in this figure are from one oocyte. For $A$ and $B$, the current traces are digital subtractions of control traces obtained in the absence of TBOA minus traces obtained in the presence of $5 \mu \mathrm{M}$ TBOA. In the sweep obtained in the presence of TBOA, the drug application was begun at the time point denoted by the triangle and persisted for the duration of the sweep. The top traces represent the voltage protocol performed in the absence and presence of TBOA. The negative potential was $-70 \mathrm{mV}$, and the positive potential was $+60 \mathrm{mV}$. $A$, Note that when TBOA was applied at $-70 \mathrm{mV}$, symmetrical TBOA-sensitive presteady-state currents were isolated by this protocol, similar to those shown in Figure $3 A . B$, When TBOA application was begun $100 \mathrm{msec}$ after the onset of the positive voltage pulse, a negative presteady-state transient of amplitude similar to that of the transient in $A$ is observed on return to $-70 \mathrm{mV}$. No positive current transient at the onset of the positive voltage pulse is observed with this protocol because at this time point TBOA was absent in both of the traces used in the subtraction.

macroscopic currents. We used the permeant anion thiocyanate as the major charge carrier for these experiments.

The on rate of current development in GLAST has been reported to be linear with glutamate concentrations through 60 $\mu \mathrm{M}$ (Wadiche and Kavanaugh, 1998). In agreement with this, we observed a nearly linear increase in the rate constant of current rise from 10 to $50 \mu \mathrm{M}$ glutamate (Fig. $5 B$ ). Although in Figure $5 B$ a linear regression was fit through $50 \mu \mathrm{M}$ glutamate, to avoid using glutamate concentrations possibly near saturation, we systematically analyzed the glutamate dependence of the rate of current development between 10 and $30 \mu \mathrm{M}$ at -70 and $+60 \mathrm{mV}$. There was a slight voltage dependence on the rate of current development at all glutamate concentrations (Fig. $5 A$ ), possibly reflecting voltage-dependent steps in transport or anion channel gating. However, the glutamate dependence of the on rate was not significantly altered by voltage (Fig. $5 B)(p>0.1 ; n=8$ patches at $10 \mu \mathrm{M}$ and 4 patches at $30 \mu \mathrm{M})$. The rate obtained by linear regression between on rates at $10 \mu \mathrm{M}$ glutamate (rate constants of $54.4 \pm 5.8 / \mathrm{sec}$ at $-70 \mathrm{mV}$ and $34.3 \pm 7.2 / \mathrm{sec}$ at $+60 \mathrm{mV} ; n=8$ patches) and $30 \mu \mathrm{M}$ glutamate $(224.6 \pm 35.7 / \mathrm{sec}$ at $-70 \mathrm{mV}$ and $174.4 \pm 57 / \mathrm{sec}$ at $+60 \mathrm{mV} ; n=4$ patches) was $9.5 \times 10^{6} \mathrm{M} / \mathrm{sec}$ at $-70 \mathrm{mV}$ and $8.7 \times 10^{6} \mathrm{M} / \mathrm{sec}$ at $+60 \mathrm{mV}$. These estimates are similar to a recent estimate at negative potentials using the human GLAST homolog (Wadiche and Kavanaugh, 1998).

Excised patches exhibited inward rectification in steady-state responses (Fig. 5A). Although the inward rectification in patches could represent fewer available transporters at positive potentials, this possibility is rendered unlikely by the presteady-state mea- 
A

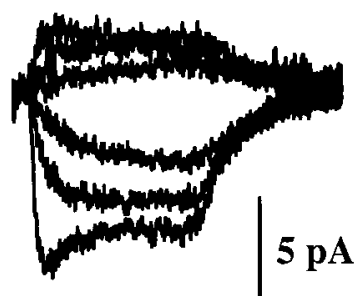

B

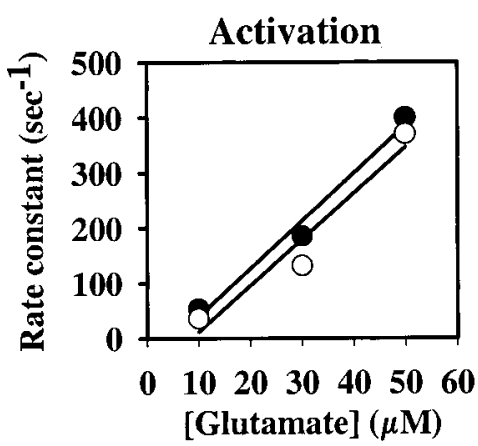

C

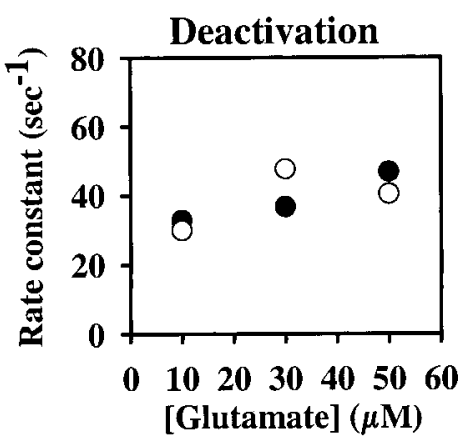

Figure 5. Neither binding rate nor deactivation rate of GLAST anion currents is detectably altered by membrane voltage in excised patches. Data in all panels are from the same patch. $A$, Exemplar traces from an outside-out patch excised from an oocyte expressing GLAST. The patch was held at $-70 \mathrm{mV}$ (inward currents) and $+60 \mathrm{mV}$ (outward currents) and rapidly exposed to 10,30 , and $50 \mu \mathrm{M}$ L-glutamate for $100 \mathrm{msec}$. The extracellular solutions contained $\mathrm{SCN}^{-}$as the primary anion. $B$, Activation rate was estimated from monoexponential fits to current rises in $A$. The solid lines are a linear regression fit to the data. Solid circles represent data at $-70 \mathrm{mV}$; open circles represent data at $+60 \mathrm{mV}$. C, Deactivation times from monoexponential fits to the off responses of glutamate currents after the $100 \mathrm{msec}$ application to the patch depicted in $A$. Solid circles represent deactivation at $-70 \mathrm{mV}$; open circles represent deactivation at $+60 \mathrm{mV}$.

surements in oocytes (Figs. $3 A, 4$ ), which suggests transporters are available to bind TBOA at positive potentials. Therefore, inward rectification in patches likely represents the slowed turnover of transporters at positive potentials and/or inherent rectification of the conductance underlying the anion current.

Deactivation rate is correlated with agonist affinity for ligandgated channels (Lester and Jahr, 1992; Jones et al., 1998). No influence of either glutamate concentration or voltage on deactivation of currents could be detected in excised patches (Fig. 5C).
Average deactivation time constant across glutamate concentrations was $36.7 \pm 5.3 \mathrm{msec}$ at $-70 \mathrm{mV}$ and $45.8 \pm 7.7 \mathrm{msec}$ at +60 $\mathrm{mV}(n=12$ patches; $p>0.1)$. The above results suggest that glutamate binding rate is not reduced by depolarization and thus that simple buffering does not underlie the prolonged EPSCs when glia are depolarized. We conclude that prevention of buffering is not the major reason that glial depolarization prolongs EPSCs.

\section{Accumulation of glutamate cannot explain prolonged EPSCs}

We next addressed the hypothesis that glutamate accumulation may cause EPSC prolongations. Accumulation of endogenous agonist might be expected to prolong EPSC decays through a cooperative action of ambient and synaptic agonist (Hartzell et al., 1975). This effect is expected to be particularly strong under conditions in which synaptic transmitter lingers longer or has larger spatial spillover, precisely the conditions under which transporter inhibition has been shown to have its strongest effects on EPSC time course (Mennerick and Zorumski, 1995; Takahashi et al., 1995).

Most transport inhibitors described, including glial membrane depolarization, should promote extracellular glutamate accumulation (Kanner and Bendahan, 1982; Kanner and Marva, 1982; Szatkowski et al., 1990; Mennerick and Zorumski, 1994). In many preparations, glutamate may accumulate from surrounding synapses in the presence of transport inhibitors (Isaacson and Nicoll, 1993; Sarantis et al., 1993; Barbour et al., 1994; Maki et al., 1994). This problem is reduced in microcultures where a solitary neuron is examined. Although the importance of glutamate efflux through transporters is controversial (Szatkowski et al., 1990; Zerangue and Kavanaugh, 1996), microcultures are potentially susceptible to glutamate release via heteroexchange in the presence of exogenously applied substrates (Kanner and Bendahan, 1982) and to reverse uptake during depolarizations or manipulation of extracellular or intracellular ions (Szatkowski et al., 1990). Our strategy was to determine whether significant heteroexchange occurs in response to substrate application and whether a nonsubstrate inhibitor, which should not promote heteroexchange, also prolongs EPSCs. For these experiments we used the nonsubstrate inhibitor TBOA (Lebrun et al., 1997; Shimamoto et al., 1998). We compared TBOA with the parent compound threo-3-hydroxyaspartate (THA) and L-trans-pyrrolidine-2,4dicarboxylate (PDC), both of which share similar potency to TBOA at the GLAST transporter but are transporter substrates (Arriza et al., 1994; Tanaka, 1994; Shimamoto et al., 1998).

To determine whether substrates induce significant efflux via heteroexchange, we used neuronal NMDA receptors in microcultures as a sensitive detector of local glutamate accumulation. Substrates and other drugs were applied to a microculture in a solution designed to potentiate NMDA receptor-mediated responses while inhibiting synaptic release of glutamate. The extracellular solution contained no added calcium or magnesium, 10 $\mu \mathrm{M}$ glycine, 1-5 $\mu \mathrm{M}$ NBQX, $25 \mu \mathrm{M}$ bicuculline, and $250 \mathrm{~nm}$ tetrodotoxin. Under these conditions, the substrates THA and PDC consistently produced APV-sensitive inward currents in microculture neurons. This inward current could be attributable to accumulating glutamate released via heteroexchange. However, we also found that at high micromolar concentrations, these substrates produced currents in excised outside-out membrane patches raised above the cell layer, confirming previous suggestions that these transporter substrates directly activate NMDA 


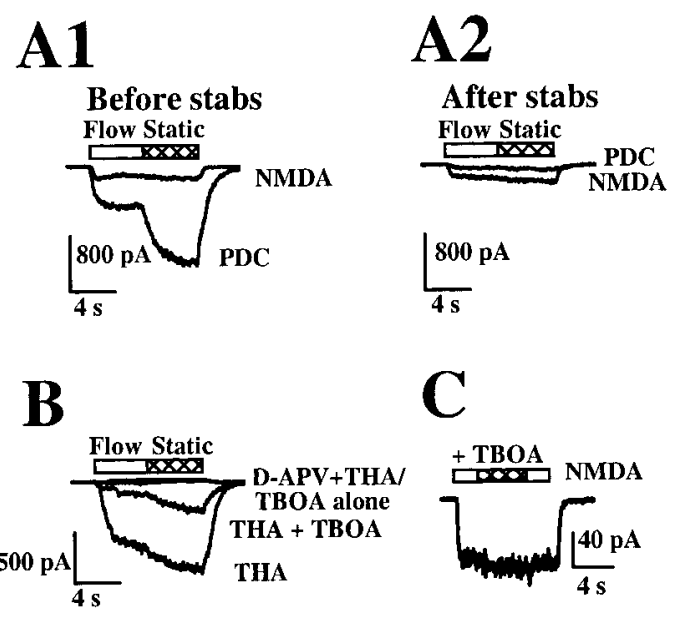

Figure 6. Evidence for heteroexchange in the presence of glutamate transporter substrates. $A 1$, The substrate PDC $(10 \mu \mathrm{M})$ causes an inward current that increases when local perfusion of the microculture is halted. The response to the nonsubstrate $N$-methyl D-aspartate receptor agonist NMDA $(3 \mu \mathrm{M})$ exhibits no increase when solution flow is halted. For these experiments, AMPA receptors were blocked with $2 \mu \mathrm{M} \mathrm{NBQX,}$ $\mathrm{Mg}^{2+}$ was removed from the bath, and $10 \mu \mathrm{M}$ glycine was added to the extracellular solution. In addition, extracellular $\mathrm{Ca}^{2+}$ was omitted from the bath to diminish synaptic glutamate release. $A 2$, After underlying glia are damaged, the response to PDC, including the enhancement under stop-flow conditions, is nearly abolished, whereas the response to NMDA is nearly unchanged. $B$, THA $(5 \mu \mathrm{M})$ causes an increased inward current with perfusion cessation similar to that observed with PDC. This response is totally abolished with addition of $100 \mu \mathrm{M}$ D-APV to the perfusion solution. TBOA $(50 \mu \mathrm{M})$ causes no current when applied alone but depresses the response to THA application. $C$, TBOA $(50 \mu \mathrm{M})$ does not detectably alter currents in response to exogenously applied NMDA $(5 \mu \mathrm{M})$.

receptors (Tong and Jahr, 1994). Because of this, we sought a way to determine whether neuronal whole-cell currents in response to substrate application are caused at least partly by heteroexchange of glutamate through transporters.

We reasoned that if accumulating endogenous glutamate explains at least part of the whole-cell NMDA receptor current, the constant perfusion typically used in our experiments might be washing away accumulating glutamate and that ceasing the perfusion should allow more accumulation. As predicted, the amplitude of the current usually increased in a PDC- or THAcontaining static bath (19 of 29 cells) (Fig. 6A,B), suggesting buildup of an endogenous substance. The current amplitude of responses to 1-3 $\mu \mathrm{M}$ NMDA, an NMDA receptor agonist without transporter activity, increased only slightly as perfusion was halted (Fig. 6A). In six cells the PDC response was potentiated $266 \pm 70 \%$, whereas NMDA responses in the same cell changed by only $43 \pm 23 \%$. The smaller potentiation during NMDAelicited responses was not caused by response saturation, because NMDA responses were always smaller than transporter substrate responses $(-35.6 \pm 8.5 \mathrm{pA}$ vs $-109.2 \pm 27 \mathrm{pA})$. The small apparent accumulation with NMDA application could be caused by accumulation of glutamate from spontaneous synaptic release or other sources.

Figure $6 A 1, A 2$ shows an example in which $10 \mu \mathrm{M}$ PDC was perfused onto a microculture neuron for $5 \mathrm{sec}$, and then solution flow was halted. We have shown previously that glia can be destroyed acutely while neural integrity is preserved in microcultures (Mennerick et al., 1998). To determine whether glia are the source of accumulating glutamate, in the case shown in Figure
$6 A 2$, the underlying glia were destroyed by mechanically rupturing the glial membrane with a sharp pipette. The response to PDC was greatly reduced, whereas the response to $3 \mu \mathrm{M}$ NMDA was preserved (Fig. 6A1, $A 2$ ). In five microcultures, the PDC response after astrocyte elimination was $46 \pm 19 \%$ of the baseline PDC response before elimination, whereas the control NMDA response after elimination was $108 \pm 17 \%$ of the baseline response. These results suggest that a significant amount of accumulation observed in response to substrate application is from microculture glia.

Response potentiation in static bath conditions was observed with both PDC and THA and was totally abolished by D-APV (Fig. 6B). In cells with a strongly potentiated static bath response, both the steady-state response during perfusion and the potentiated static-bath current were diminished by coapplication of TBOA (Fig. 6B). Average inhibition by $50 \mu \mathrm{M}$ TBOA of the static-bath response was $49 \pm 9 \%$ in seven cells treated with either $5 \mu \mathrm{M}$ THA or $5 \mu \mathrm{M}$ PDC. The inhibition by TBOA suggests that the accumulation of agonist is largely caused by TBOAsensitive heteroexchange rather than accumulation of glutamate from another source.

TBOA $(50 \mu \mathrm{M})$ alone had no effect on NMDA-induced responses (Fig. 6C) and had either no effect on baseline membrane currents (Fig. $6 B$ ) or induced a small inward current in stop-flow conditions (four of eight neurons, $53.6 \pm 24.6 \mathrm{pA}$ ). This result suggests sources of glutamate accumulation other than heteroexchange or a weak agonist effect of TBOA. Similar glutamate receptor activation in the presence of TBOA has recently been reported and preliminarily attributed to a cystine/glutamate exchanger (Jabaudon et al., 1999).

These results suggest that glutamate accumulation is significant during application of transporter substrates, and previous results suggest that glial depolarization can promote glutamate release via reverse uptake (Mennerick and Zorumski, 1994). Therefore, we used a comparison of THA and TBOA effects on EPSCs to determine whether accumulating glutamate might explain EPSC prolongations. TBOA and its parent compound THA produced similar prolongations of AMPA receptor-mediated EPSCs when used at equimolar concentrations in the presence of $10 \mu \mathrm{M} \mathrm{cy-}$ clothiazide (Fig. 7). On average, THA prolonged EPSC 10-90\% decay time by $41 \pm 9 \%$, and TBOA prolonged EPSCs by $57 \pm$ $17 \%$ in the same cells. Examination of THA and TBOA effects in individual cells showed a high correlation between the effects of the two drugs (Fig. 7B), suggesting that THA and TBOA prolong EPSCs through a similar mechanism.

It is still possible that glutamate accumulation is promoted in the presence of TBOA, as suggested by inward current shifts in some cells. Therefore, we asked whether low concentrations of glutamate mimic EPSC prolongations observed in the presence of transport inhibitors. Figure $8 A$ shows the effect on an EPSC (no CYZ present) of applying $2.5 \mu \mathrm{M} \mathrm{L}$-glutamate and $50 \mu \mathrm{M}$ PDC. PDC caused a small inward shift in the baseline holding current $(-12 \mathrm{pA})$ but no decrease in the peak amplitude of the EPSC (Fig. $8 A$, inset). In contrast, application of $2.5 \mu \mathrm{M}$ glutamate caused a larger inward current $(-50 \mathrm{pA})$ and slight suppression of the EPSC peak amplitude, indicating that exogenous glutamate reached synaptic regions in excess of endogenous glutamate concentrations achieved during PDC application. Suppression of the EPSC was $22 \pm 3 \%$ by $2.5 \mu \mathrm{M}$ glutamate and $2 \pm 4 \%$ by $50 \mu \mathrm{M}$ PDC ( $n=10 ; p<0.05$, paired $t$ test). Prolongation of the EPSC was more pronounced in the presence of PDC than in the presence of glutamate (Fig. 8). In 10 cells, the 10-90\% decay time was 
A
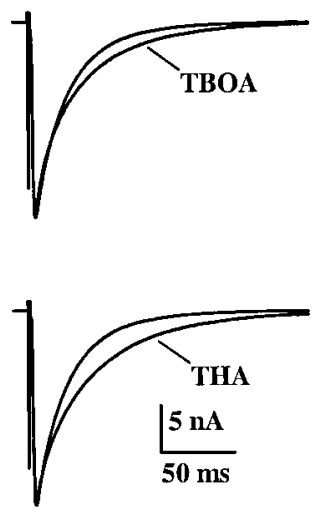

B

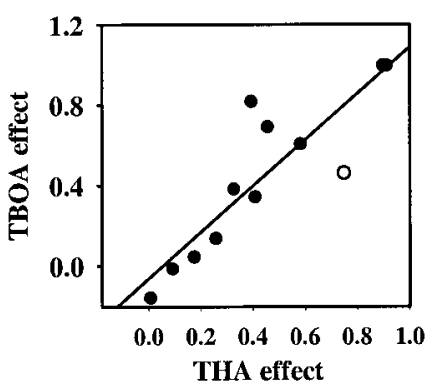

Figure 7. TBOA and THA have similar effects on AMPA receptormediated synaptic currents. $A$, Autaptic currents in the presence of $10 \mu \mathrm{M}$ cyclothiazide are prolonged by TBOA $(50 \mu \mathrm{M})$ and THA $(50 \mu \mathrm{M}) . B$, Plot of the effect of TBOA $(50 \mu \mathrm{M})$ versus the effect of THA $(50 \mu \mathrm{M})$ on the $10-90 \%$ decay times of synaptic currents in individual cells. Both axes on the graph represent the fractional increase in the $10-90 \%$ decay time of

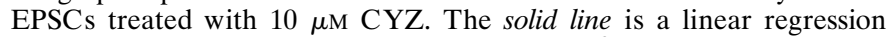
through the points, with a slope of 1.15 and with $r^{2}=0.78$. The open circle represents the example shown in $A$. For these experiments, $50 \mu \mathrm{M}$ D-APV was included in the bath solution to block NMDA receptors.

prolonged by $21 \pm 8 \%$ with PDC and $-1 \pm 5 \%$ by glutamate $(p<0.05$, paired $t$ test). Taken together, the results indicate that glutamate at levels likely to accumulate during uptake inhibition in microcultures is unlikely to account for the EPSC prolongations observed. The results also suggest that properties of AMPA receptors, including low affinity for glutamate (Patneau and Mayer, 1990) and profound desensitization (Trussell et al., 1988), help limit the cooperativity observed between exogenous and synaptic transmitters observed at other synapses (Hartzell et al., 1975).

As a positive control, we tested whether under extreme conditions, glutamate accumulation itself can indeed prolong EPSCs through a cooperative interaction with synaptic glutamate. We examined the effect of exogenous glutamate on EPSCs in lithium solutions to block uptake and in the presence of $10 \mu \mathrm{M} \mathrm{CYZ}$ to diminish AMPA receptor desensitization. Under these conditions, applications of $2.5 \mu \mathrm{M}$ glutamate prolonged EPSCs slightly (Fig. $8 B$ ), indicating that with transport and receptor desensitization blocked, a cooperative interaction between ambient and synaptic agonist occurs under these extreme experimental conditions. Three of six neurons treated with $2.5 \mu \mathrm{M}$ glutamate in $\mathrm{Li}^{+}$
A

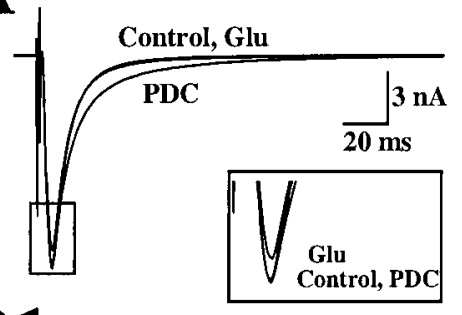

B1

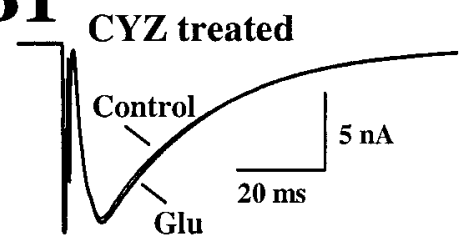

B2

CYZ treated $+\mathrm{Li}^{+}$
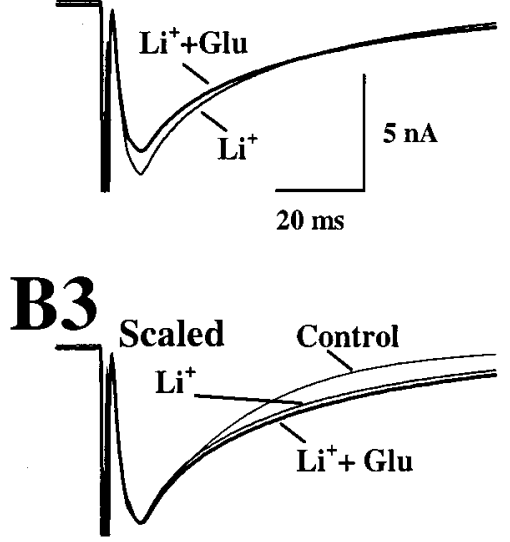

Figure 8. Effect of ambient glutamate on EPSC time course. A, An EPSC is prolonged by the application of $50 \mu \mathrm{M}$ PDC. In the same cell, $2.5 \mu \mathrm{M}$ glutamate did not prolong the EPSC. The inset shows a magnified view of the peak EPSC responses. PDC did not affect the peak EPSC, whereas glutamate slightly depressed the peak amplitude, suggesting that ambient glutamate in this condition reached higher levels than during PDC application. B1, An EPSC in $10 \mu \mathrm{M} \mathrm{CYZ}$ is not affected by the application of $2.5 \mu \mathrm{M}$ L-glutamate [Control, thin trace; glutamate $(G l u)$, thick trace]. B2,

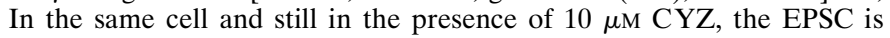
prolonged by substitution of lithium for sodium in the bath solution. In the presence of lithium, glutamate causes a detectable inward current and depresses the peak amplitude of the EPSC (baseline lithium, thin trace; glutamate, thick trace). B3, The traces from $B 2$ and the control trace from $B 1$ have been offset and scaled to show the prolongation induced by $\mathrm{Li}^{+}$ alone and the slight additional prolongation of the EPSC by glutamate delivered in the $\mathrm{Li}^{+}$saline. For all experiments, AMPA-receptor EPSCs were isolated by including $50 \mu \mathrm{M}$ D-APV in the bath solution.

responded with reliable, reversible EPSC prolongations similar to the cell shown in Figure 8. We conclude that although glutamate accumulation may participate in prolonged EPSCs, blockade of glutamate transporters accounts most strongly for EPSC prolongations in the presence of uptake inhibitors.

\section{DISCUSSION}

Glutamate transport inhibitors prolong EPSCs at various synapses in vitro and in situ. Our results suggest that neither a glutamate buffering effect nor accumulation of ambient glutamate explains the prolongation of EPSCs caused by glutamate transporter inhibitors and substrates. We observe a slight increase in 
apparent affinity of transporters for glutamate at positive membrane potentials; therefore, simple binding of glutamate is not sufficient to explain the role of transporters in truncating synaptic glutamate transients. In addition, although we have shown that accumulation of glutamate occurs with application of transporter substrates, accumulation of glutamate via heteroexchange or reverse uptake cannot explain EPSC prolongations, because the nonsubstrate inhibitor TBOA does not promote glutamate efflux but has effects similar to the substrate THA on EPSC time course. Also, exogenous glutamate applications do not mimic effects of transporter substrates. Our results do not address the very rapid effects of transporter substrates observed by others (Tong and Jahr, 1994; Diamond and Jahr, 1997), whereby the peak concentration of glutamate achieved is potentiated by inhibition of transporters. It is therefore likely that these rapid effects, evidenced by changes in the rise time and peak of the EPSC at physiological temperatures, are entirely consistent with the present observations. In this case a stationary buffer property of transporters functions on the fastest time scales, and translocation functions on the slower time scale of EPSC decay.

For glutamate turnover to explain the EPSC prolongations observed, transporters must be present at high density near synaptic sites, or turnover must be substantially faster than suggested. It is possible that both propositions are accurate. Recent physiological and anatomical results suggest that in culture and in situ transporter density are very high, approaching 11,000 transporters per $\mu \mathrm{m}^{-2}$ in CA1 stratum radiatum of the rat hippocampus (Lehre and Danbolt, 1998). Alternative methods for calculating substrate turnover have also recently suggested a somewhat faster turnover rate than previous techniques: $\sim 40 / \mathrm{sec}$ at $24^{\circ} \mathrm{C}$ (Bergles and Jahr, 1998). These observations also suggest that the glial transporter-associated currents recorded during synaptic transmission (Fig. 1) represent significant glutamate translocation on the time scale of an EPSC.

Our results suggest that the GLAST glutamate transporter and the transporters responsible for glutamate uptake in microculture astrocytes (largely GLAST) (Mennerick et al., 1998) do not change their affinity for glutamate with changes in membrane potential. Other steps in the transport cycle, such as sodium binding or closely related steps, may impart the observed voltage dependence of transport (Brew and Attwell, 1987). However, it is noteworthy that if, as suggested, voltage alters only steps in the translocation of glutamate, membrane potential must decrease the probability that a molecule of glutamate transports rather than unbinds. In this case, the meaning of buffering becomes more complicated, and it is precisely because glutamate can dissociate from the transporter (the transporter no longer effectively buffers glutamate) that EPSCs are prolonged.

A particularly surprising finding of the present results is that the nonsubstrate inhibitor TBOA binds equally well to the transporter at positive potentials and at negative potentials (Figs. 3, 4). In current models of the transporter, sodium must bind to the extracellular-facing transporter before glutamate or glutamate analogs can bind the transporter (Kanner and Bendahan, 1982; Wadiche et al., 1995b; Otis and Jahr, 1998). It is also generally believed that presteady-state currents represent the binding (with hyperpolarization) and unbinding (with depolarization) of sodium at a site on the transporter at least partway through the transmembrane electric field (Wadiche et al., 1995b). The results of Figure 4 suggest either that sodium binding is not necessary for TBOA binding to the transporter or sodium binding and unbind- ing is not the basis for the presteady-state charge movements. Further work will be needed to distinguish these possibilities.

The evidence for significant glutamate efflux via heteroexchange and reverse transport is controversial. The current work shows clear evidence for rapid heteroexchange of substrates for endogenous glutamate in native cells and shows that glutamate accumulation through this mechanism can be substantial (Volterra et al., 1996). Although accumulation of synaptic glutamate released from surrounding synapses may also be a source of accumulated glutamate in many preparations, in our studies this route of accumulation is minimized by restricting analysis to solitary neurons and by removing extracellular calcium to diminish synaptic glutamate release.

Three additional lines of evidence suggest that the glutamate accumulation observed in the present study occurs via heteroexchange. First, the nonsubstrate inhibitor TBOA does not induce significant accumulation currents in neurons at concentrations 5to 10 -fold higher than substrates. Second, eliminating underlying glia nearly eliminates the substrate-induced currents. Finally, TBOA diminishes the accumulation currents induced by substrates.

The present results suggest that although ambient glutamate is not likely to be a large contributor to prolonged EPSCs, efflux of glutamate through astrocyte transporters is significant. Ambient transmitter accumulation may be expected to interact cooperatively at postsynaptic receptors with synaptic transmitter at time points during the EPSC when receptors are not saturated (Hartzell et al., 1975). These time points are likely to be late in the EPSC when glutamate levels have dropped or have diff used beyond the synapse, precisely the time points affected by transport inhibitors (Mennerick and Zorumski, 1995; Takahashi et al., 1995; Otis et al., 1996). Our results rule out an important contribution of ambient glutamate to the time course of individual EPSCs, although a small cooperative effect of glutamate was observed with transporters blocked and with receptors sensitized with CYZ. It is likely that under normal conditions the low affinity of AMPA receptors (Patneau and Mayer, 1990) and their desensitization to low glutamate concentrations (Trussell and Fischbach, 1989) limit the cooperative interaction between ambient and synaptic transmitter.

Despite the lack of effect of glutamate accumulation on the time course of AMPA receptor-mediated EPSCs, it is clear that glutamate released from astrocytes in the presence of transport substrates can activate neuronal NMDA receptors, and previous work has suggested that substrates can depress transmission through glutamate accumulation (Maki et al., 1994). Other pathological or physiological conditions under which efflux might occur and the contribution of this efflux to modulation of neurotransmission or initiation of neurotoxicity await further study.

\section{REFERENCES}

Arriza JL, Fairman WA, Wadiche JI, Murdoch GH, Kavanaugh MP, Amara SG (1994) Functional comparisons of three glutamate transporter subtypes cloned from human motor cortex. J Neurosci 14:5559-5569.

Barbour B, Brew H, Attwell D (1991) Electrogenic uptake of glutamate and aspartate into glial cells isolated from the salamander (Ambystoma) retina. J Physiol (Lond) 436:169-193.

Barbour B, Keller BU, Llano I, Marty A (1994) Prolonged presence of glutamate during excitatory synaptic transmission to cerebellar Purkinje cells. Neuron 12:1331-1343.

Bergles DE, Jahr CE (1998) Glial contribution to glutamate uptake at schaffer collateral-commissural synapses in the hippocampus. J Neurosci 18:7709-7716. 
Billups B, Rossi D, Attwell D (1996) Anion conductance behavior of the glutamate uptake carrier in salamander retinal glial cells. J Neurosci 16:6722-6731.

Brew H, Attwell D (1987) Electrogenic glutamate uptake is a major current carrier in the membrane of axolotl retinal glial cells. Nature 327:707-709.

Colquhoun D (1998) Binding, gating, affinity and efficacy: the interpretation of structure-activity relationships for agonists and of the effects of mutating receptors. Br J Pharmacol 125:924-947.

Diamond JS, Jahr CE (1995) Asynchronous release of synaptic vesicles determines the time course of the AMPA receptor-mediated EPSC. Neuron 15:1097-1107.

Diamond JS, Jahr CE (1997) Transporters buffer synaptically released glutamate on a submillisecond time scale. J Neurosci 17:4672-4687.

Eliasof S, Jahr CE (1996) Retinal glial cell glutamate transporter is coupled to an anionic conductance. Proc Natl Acad Sci USA 93:4153-4158.

Hartzell HC, Kuffler SW, Yoshikami D (1975) Post-synaptic potentiation: interaction between quanta of acetylcholine at the skeletal neuromuscular synapse. J Physiol (Lond) 251:427-463.

Isaacson JS, Nicoll RA (1993) The uptake inhibitor L-trans-PDC enhances responses to glutamate but fails to alter the kinetics of excitatory synaptic currents in the hippocampus. J Neurophysiol 70:2187-2191.

Jabaudon D, Shimamoto K, Yasuda-Kamatani Y, Scanziani M, Gahwiler BH, Gerber U (1999) Inhibition of uptake unmasks rapid extracellular turnover of glutamate of nonvesicular origin. Proc Natl Acad Sci USA 96:8733-8788.

Jones MV, Sahara Y, Dzubay JA, Westbrook GL (1998) Defining affinity wth the $\mathrm{GABA}_{\mathrm{A}}$ receptor. J Neurosci 18:8590-8604.

Kanner BI, Bendahan A (1982) Binding order of substrates to the sodium and potassium ion coupled L-glutamic acid transporter from rat brain. Biochemistry 21:6327-6330.

Kanner BI, Marva E (1982) Efflux of L-glutamate by synaptic plasma membrane vesicles isolated from rat brain. Biochemistry 21:3143-3147.

Kinney GA, Overstreet LS, Slater NT (1997) Prolonged physiological entrapment of glutamate in the synaptic cleft of cerebellar unipolar brush cells. J Neurophysiol 78:1320-1333.

Lavoie AM, Twyman RE (1996) Direct evidence for diazepam modulation of GABAA receptor microscopic affinity. Neuropharmacology 35:1383-1392.

Lebrun B, Sakaitani M, Shimamoto K, Yasuda-Kamatani Y, Nakajima T (1997) New beta-hydroxyaspartate derivatives are competitive blockers for the bovine glutamate/aspartate transporter. J Biol Chem 272:20336-20339.

Lehre KP, Danbolt NC (1998) The number of glutamate transporter subtype molecules at glutamatergic synapses: chemical and stereological quantification in young adult rat brain. J Neurosci 18:8751-8757.

Lester HA, Mager S, Quick MW, Corey JL (1994) Permeation properties of neurotransmitter transporters. Annu Rev Pharmocol Toxicol 34:219-249.

Lester RA, Jahr CE (1992) NMDA channel behavior depends on agonist affinity. J Neurosci 12:635-643.

Maki R, Robinson MB, Dichter MA (1994) The glutamate uptake inhibitor L-trans-pyrrolidine-2,4- dicarboxylate depresses excitatory synaptic transmission via a presynaptic mechanism in cultured hippocampal neurons. J Neurosci 14:6754-6762.

Mennerick S, Zorumski CF (1994) Glial contributions to excitatory neurotransmission in cultured hippocampal cells. Nature 368:59-62.

Mennerick S, Zorumski CF (1995) Presynaptic influence on the time course of fast excitatory synaptic currents in cultured hippocampal cells. J Neurosci 15:3178-3192.
Mennerick S, Que J, Benz A, Zorumski CF (1995) Passive and synaptic properties of neurons grown in microcultures and in mass cultures. J Neurophysiol 73:320-332.

Mennerick S, Dhond RP, Benz A, Xu W, Rothstein JD, Danbolt NC, Isenberg KE, Zorumski CF (1998) Neuronal expression of the glutamate transporter GLT-1 in hippocampal microcultures. J Neurosci 18:4490-4499.

Otis TS, Jahr CE (1998) Anion currents and predicted glutamate flux through a neuronal glutamate transporter. J Neurosci 18:7099-7110.

Otis TS, Wu Y-C, Trussell LO (1996) Delayed clearance of transmitter and the role of glutamate transporters at synapses with multiple release sites. J Neurosci 16:1634-1644.

Parent L, Supplisson S, Loo DDF, Wright EM (1992) Electrogenic properties of the cloned $\mathrm{Na}^{+} /$glucose cotransporter. I. Voltage-clamp studies. J Membr Biol 125:49-62.

Patneau DK, Mayer ML (1990) Structure-activity relationships for amino acid transmitter candidates acting at $N$-methyl-D-aspartate and quisqualate receptors. J Neurosci 10:2385-2399.

Sarantis M, Ballerini L, Miller B, Silver RA, Edwards M, Attwell D (1993) Glutamate uptake from the synaptic cleft does not shape the decay of the non-NMDA component of the synaptic current. Neuron 11:541-549.

Seal RP, Amara SG (1998) A reentrant loop domain in the glutamate carrier EAAT1 participates in substrate binding and translocation. Neuron 21:1487-1498.

Shih TM, Smith RD, Toro L, Goldin AL (1998) High-level expression and detection of ion channels in Xenopus oocytes. Methods Enzymol 293:529-556.

Shimamoto K, Lebrun B, Yasuda-Kamatani Y, Sakaitani M, Shigeri Y, Yumoto N, Nakajima T (1998) DL-threo-beta-benzyloxyaspartate, a potent blocker of excitatory amino acid transporters. Mol Pharmacol 53:195-201.

Szatkowski M, Barbour B, Attwell D (1990) Non-vesicular release of glutamate from glial cells by reversed electrogenic glutamate uptake. Nature 348:443-446.

Takahashi M, Kovalchuk Y, Attwell D (1995) Presynaptic and postsynaptic determinants of EPSC waveform at cerebellar climbing fiber and parallel fiber to Purkinje cell synapses. J Neurosci 15:5693-5702.

Tanaka K (1994) Pharmacological characterization of a cloned rat glutamate transporter (GluT-1). Brain Res Mol Brain Res 21:167-170.

Tong G, Jahr CE (1994) Block of glutamate transporters potentiates postsynaptic excitation. Neuron 13:1195-1203.

Trussell LO, Fischbach GD (1989) Glutamate receptor desensitization and its role in synaptic transmission. Neuron 3:209-218.

Trussell LO, Thio LL, Zorumski CF, Fischbach GD (1988) Rapid desensitization of glutamate receptors in vertebrate central neurons. Proc Natl Acad Sci USA 85:4562-4566.

Volterra A, Bezzi P, Rizzini BL, Trotti D, Ullensvang K, Danbolt NC, Racagni G (1996) The competitive transport inhibitor L-transpyrrolidine-2, 4-dicarboxylate triggers excitotoxicity in rat cortical neuron-astrocyte co-cultures via glutamate release rather than uptake inhibition. Eur J Neurosci 8:2019-2028.

Wadiche JI, Kavanaugh MP (1998) Macroscopic and microscopic properties of a cloned glutamate transporter chloride channel. J Neurosci 18:7650-7661.

Wadiche JI, Amara SG, Kavanaugh MP (1995a) Ion fluxes associated with excitatory amino acid transport. Neuron 15:721-728.

Wadiche JI, Arriza JL, Amara SG, Kavanaugh MP (1995b) Kinetics of a human glutamate transporter. Neuron 14:1019-1027.

Zerangue N, Kavanaugh MP (1996) Flux coupling in a neuronal glutamate transporter. Nature 383:634-637. 